\title{
Motion Compensation Strategies in Magnetic Resonance Imaging
}

\author{
Ruud B. van Heeswijk, ${ }^{a, b}$ Gabriele Bonanno, ${ }^{a, b}$ Simone Coppo, ${ }^{a, b}$ Andrew Coristine, ${ }^{a, b}$ \\ Tobias Kober, ${ }^{\mathrm{c}}$ \& Matthias Stuber ${ }^{\mathrm{a}, \mathrm{b}}$
}

aDepartment of Radiology, Centre Hospitalier Universitaire Vaudois and University of Lausanne, Lausanne, Switzerland; ' Center for Biomedical Imaging (CIBM), Lausanne, Switzerland; 'Advanced Clinical Imaging Technology, Siemens Healthcare Sector IM\&WS S, Lausanne, Switzerland

\footnotetext{
* Address all correspondence to: Corresponding author and address: Ruud B. van Heeswijk, CHUV-CIBM, Rue de Bugnon 46 BH8.84, 1011 Lausanne, Switzerland, ruud.mri@gmail.com
}

\begin{abstract}
Image quality in magnetic resonance imaging (MRI) is considerably affected by motion. Therefore, motion is one of the most common sources of artifacts in contemporary cardiovascular MRI. Such artifacts in turn may easily lead to misinterpretations in the images and a subsequent loss in diagnostic quality. Hence, there is considerable research interest in strategies that help to overcome these limitations at minimal cost in time, spatial resolution, temporal resolution, and signal-to-noise ratio. This review summarizes and discusses the three principal sources of motion: the beating heart, the breathing lungs, and bulk patient movement. This is followed by a comprehensive overview of commonly used compensation strategies for these different types of motion. Finally, a summary and an outlook are provided.
\end{abstract}

KEY WORDS: magnetic resonance imaging, motion correction, gating, triggering, navigators

\begin{abstract}
ABBREVIATIONS: 1D: one-dimensional; 2D: two-dimensional;3D: three-dimensional; AP: anterior-posterior; bSSFP: balanced steady state free precession; CS: compressed sensing/compressive sampling; ECG: electrocardiogram; FID: free induction decay; GW: gating window; KCL: k-space centerline; LAD: left anterior descending coronary artery; LV: left ventricle; MRA: magnetic resonance angiography; MRI: magnetic resonance imaging; NAV: navigator; ONAV: orbital navigator; PROPELLER: periodically rotated overlapping parallel lines with enhanced reconstruction; RCA: right coronary artery; RF: radiofrequency; RV: right ventricle; SI: superior-inferior; SNR: signal-to-noise ratio
\end{abstract}

\section{THE EFFECT OF MOTION ON MRI}

\section{A. General Considerations}

Magnetic resonance imaging (MRI) as a medical imaging modality is unique since it is noninvasive, safe, operates without ionizing radiation, and has high soft-tissue contrast. Unfortunately, to obtain adequate image quality, the duration of image acquisition may be relatively long. This is often in conflict with patient motion. Motion leads to blurring in the images and results in discontinuities of data in the Fourier domain (k-space), which leads to additional image artifacts. This is not only detrimental to image quality, but also affects the diagnostic quality of MRI. Therefore, there is substantial need to compensate for motion in MRI. To avoid adverse effects of motion on image quality, the content of an imaged volume should be rigid and motionless at the time of image data acquisition. Simultaneously, if the image data collection is not continuous, but rather subdivided into multiple acquisitions or $\mathrm{k}$ space segments acquired at different time points, it is necessary that the imaged object always be in the same position at the time of data collection. However, these two conditions are not always easily met in vivo. Motion compensation techniques, therefore, adjust image acquisition strategies to satisfy these conditions. Motion compensation approaches that affect MRI data collection or reconstruction are termed either prospective or retrospective. Using prospective techniques, a physiological trigger 
signal that is characteristic of a periodic event (for example, the R-wave of the ECG or the diaphragm position) is used to not only initiate image data collection, but also to modify it to a certain extent. For example, if the displacement of the object relative to the coordinates of the imaged slice is known, a real-time adaptation of the slice position occurs. In contrast, and using retrospective approaches, data acquisition can no longer be influenced directly, but sophisticated image reconstruction algorithms are used to either reorder k-space segments or to account for object motion. Prospective strategies, although powerful, often suffer from time-efficiency limitations since time is lost through waiting for the trigger signal. Using retrospective strategies, such inefficiency can be avoided to a large degree, but image data acquisition can no longer be influenced, and correction schemes may introduce additional image artifacts that have to be considered.

For in vivo MRI, motion can be divided into three main categories: cardiac motion, respiratory motion, and bulk motion. The two former components are periodic, substantial, and relatively predictable, while their suppression is important for thoracic (e.g., the heart and lungs) and abdominal (e.g., the kidneys, liver, and intestines) imaging. The latter, meanwhile, is usually a one-time event that occurs randomly and in an unpredictable pattern. While it affects all in vivo MRI, it is of particular concern for brain imaging.

Since the aforementioned types of motion have quite different origins and require individual compensation strategies, we will first discuss the origins of motion before motion compensation strategies are introduced. Supporting measures aimed at minimizing the effects of motion will then be discussed and are followed by a summary.

\section{B. The Beating Heart}

The motion of the heart has a cyclically repeating pattern of contraction and relaxation. At rest, the heart beats between 50 and 100 times per minute ${ }^{1}$ this is normally the fastest movement that MRI has to account for. If not compensated for, it creates significant motion artifacts that lead to cardiac MR images with limited diagnostic value. Each heartbeat or cardiac cycle is divided in two phases: systole and diastole. Systole consists of the contraction of the heart muscle, while diastole consists of the relaxation of the heart muscle and the subsequent rest period, of which the length depends on the heart rate. If the heart rate increases, it is mainly diastole that shortens; the duration of systole remains almost unchanged. During the cardiac cycle, there are two periods of minimal heart motion: one at end systole and one during mid-diastole. These periods are commonly preferred for anatomical MR imaging of the heart.

The contraction of the heart consists of several major components that occur almost simultaneously: a shortening of both the left and right ventricles (LV and RV) in the long axis direction, a circumferential shortening of the ventricles and wall thickening, and a "wringing" motion of the $\mathrm{LV}$, which consists of a clockwise rotation at its base and a simultaneous counterclockwise rotation at its apex ${ }^{2}$ (Fig. 1). Such motion can accurately be quantified using advanced myocardial tagging 3-5 techniques, which label the magnetization (tagging) of the myocardium with a line or grid pattern prior to imaging at multiple time points in the cardiac cycle. Several studies have been performed to assess the left ventricular velocities and resulted in a maximum longitudinal velocity of $140 \pm 40$ $\mathrm{mm} / \mathrm{s},{ }^{6}$ and a maximum radial velocity of $51 \pm 18$ $\mathrm{mm} / \mathrm{s} .^{7,8}$

The specific motion of the coronary arteries has been studied as well (Fig. 2), mainly to determine when in the cardiac cycle coronary motion is minimal, and for how long this period lasts. Wang et al. ${ }^{9}$ demonstrated that the duration of this period varies from 66 to $333 \mathrm{~ms}$ and decreases with higher heart rates. Moreover, they found that the right coronary artery (RCA) moves more than twice as much as the left coronary arteries, while also reaching higher velocities. Because coronaries do not move uniformly, Johnson et al. ${ }^{10}$ focused their work on studying the motion of individual coronary segments. They noted that there is a diastolic window of from 71 to $76 \%$ of the cardiac cycle during which the ensemble of all the coronaries 


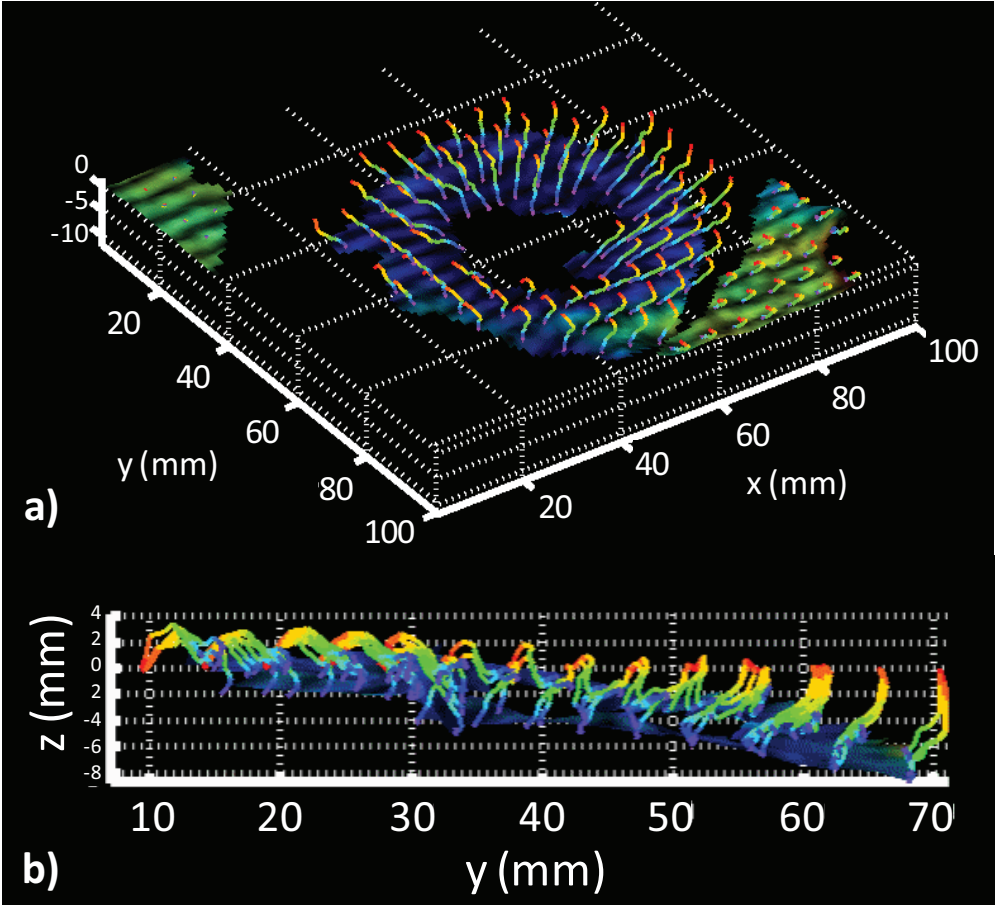

FIGURE 1: Illustration of the complex contraction and relaxation pattern of the heart. (a) Three-dimensional displacement of an equatorial cardiac short-axis slice with zHARP imaging and analysis. The slice itself (with the myocardium in the center) is displayed in its position at end systole, which is approximately $350 \mathrm{~ms}$ after the R-wave, and demonstrates maximum circumferential contraction. Lines represent the trajectory of pooled material points through time, while the colored segments indicate the incremental displacement during different cardiac phases. (b) The same slice as seen from the side, displaying the extent of through-plane motion. (Adapted and reprinted with permission from Ref. 118.)
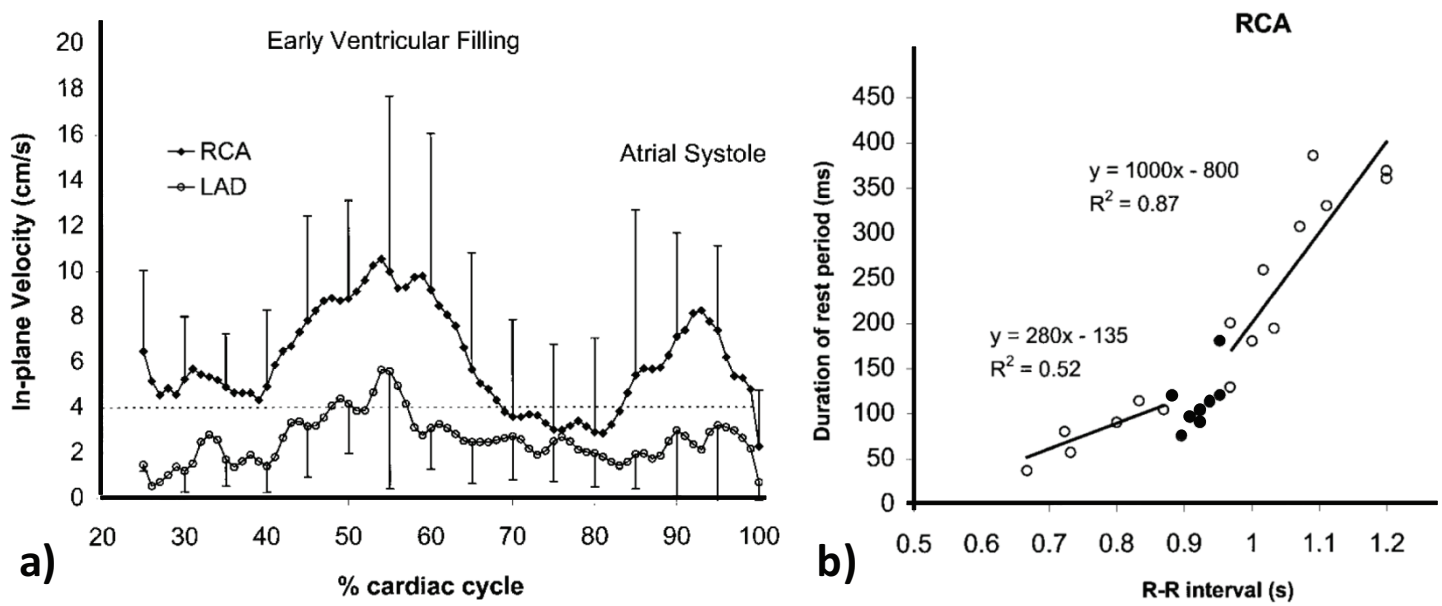

FIGURE 2: Assessment of coronary artery velocity and the relation of its rest period to the RR-interval duration. (a) In-plane diastolic velocities ( $\mathrm{cm} / \mathrm{s}$, mean \pm standard deviation) of the proximal right (RCA) and left (LAD) coronary arteries of 25 subjects. The time in the cardiac cycle is shown as percentage of the RR interval. The curves are interpolated to provide increased temporal resolution. (b) The relationships between the duration of the RCA rest period (ms) and the RR interval (s). The lines are least-squares linear regression fits for RR intervals $<0.87 \mathrm{~s}$ and $>0.97 \mathrm{~s}$, respectively. (Adapted and reprinted with permission from Ref. 20.) 
has the smallest range of movement. Knowing the length and temporal position of the diastolic rest period is thus essential for the optimization of high-resolution cardiac and coronary MRI.

\section{The Breathing Lungs}

The respiratory cycle lasts around $4-5 \mathrm{~s}$ at rest, and characterizes the tidal movement of respiratory structures such as chest wall and diaphragm. The movement of both these structures directly affects thoracic and abdominal motion, and because of the magnitude of this displacement, respiratory motion is one of the major challenges in cardiac and abdominal MRI; even more so in coronary or scar MRI, where higher spatial resolution is required. We will therefore focus this section on describing the influences of respiratory motion on that of the heart.

Wang et al. ${ }^{11}$ reported that the respiratory motion of the heart approximates a global translation in the superior-inferior (SI) direction, which is linearly related to the SI motion of the diaphragm. Since coronary imaging is one of the imaging techniques that is mostly affected by respiratory motion because of its need for high spatial resolution, specific correction factors were estimated for the RCA and the left anterior descending (LAD) coronary artery, which were 0.57 and 0.7 , respectively, although these values were found to vary widely among subjects. ${ }^{12}$ A later study by Nehrke et al. ${ }^{13}$ found a similar correlation in SI direction between the dome of the right hemidiaphragm and the left ventricle for four volunteers. However, besides confirming a linear relationship and correction factors (0.46-0.99), hysteretic loops (i.e., a lagging effect) with different paths for inspiration and expiration were also reported (Fig. 3).

These results thus indicate that a certain diaphragmatic position in the middle of a specific respiratory phase can correspond to two different heart positions. Moreover, the same counterclockwise advancement through the hysteretic loop was observed in all volunteers, which corresponds with a time lag of the respiratory-induced motion of the heart relative to that of the diaphragm. This was at- tributed both to different muscles that contract during respiration and to the viscoelastic properties of the lungs. The hysteretic effect may thus have serious consequences for the accuracy and reliability of gating and motion-correction schemes for cardiac MRI.

Results obtained in the anterior-posterior (AP) direction, i.e., between the chest and heart, show the same hysteretic behavior, but in a less pronounced manner. A study of respiratory-induced motion of the heart using rigid and nonrigid transformation parameters generated at various breathhold positions was carried out by McLeish et al. ${ }^{14}$ They demonstrated that the largest component of motion is in the inferior direction during inspiration, while the range of diaphragm displacement is up to $40 \mathrm{~mm}$ with a large standard deviation, which suggests a high intersubject variability. However, much smaller values were found when applying the same transformations to free-breathing ${ }^{15}$ data, suggesting that the breath-hold positions that were used exceed the range of normal tidal breathing or that there was residual diaphragmatic motion during acquisition. ${ }^{16}$

\section{Bulk Motion}

Bulk motion consists of the rigid translation and rotation of the part of the body that is being imaged, and is mostly caused by voluntary or involuntary patient movement. For MRI of most parts of the anatomy, it is avoided purely by patient cooperation, while anesthesia can be applied to pediatric or uncooperative patients. The patient is often assisted in avoiding motion by restriction of the movement of the imaged anatomy through cushioning or by being placed into a RF coil with a shape that inhibits movement. However, in the case of high spatial resolution imaging sequences with a long duration or in certain patients, this level of cooperation might not easily be possible and, therefore, compensation strategies for such bulk motion are necessary. The main difference between bulk motion and respiratory or cardiac motion is therefore that the movement is not necessarily periodic in nature and both its timing and 

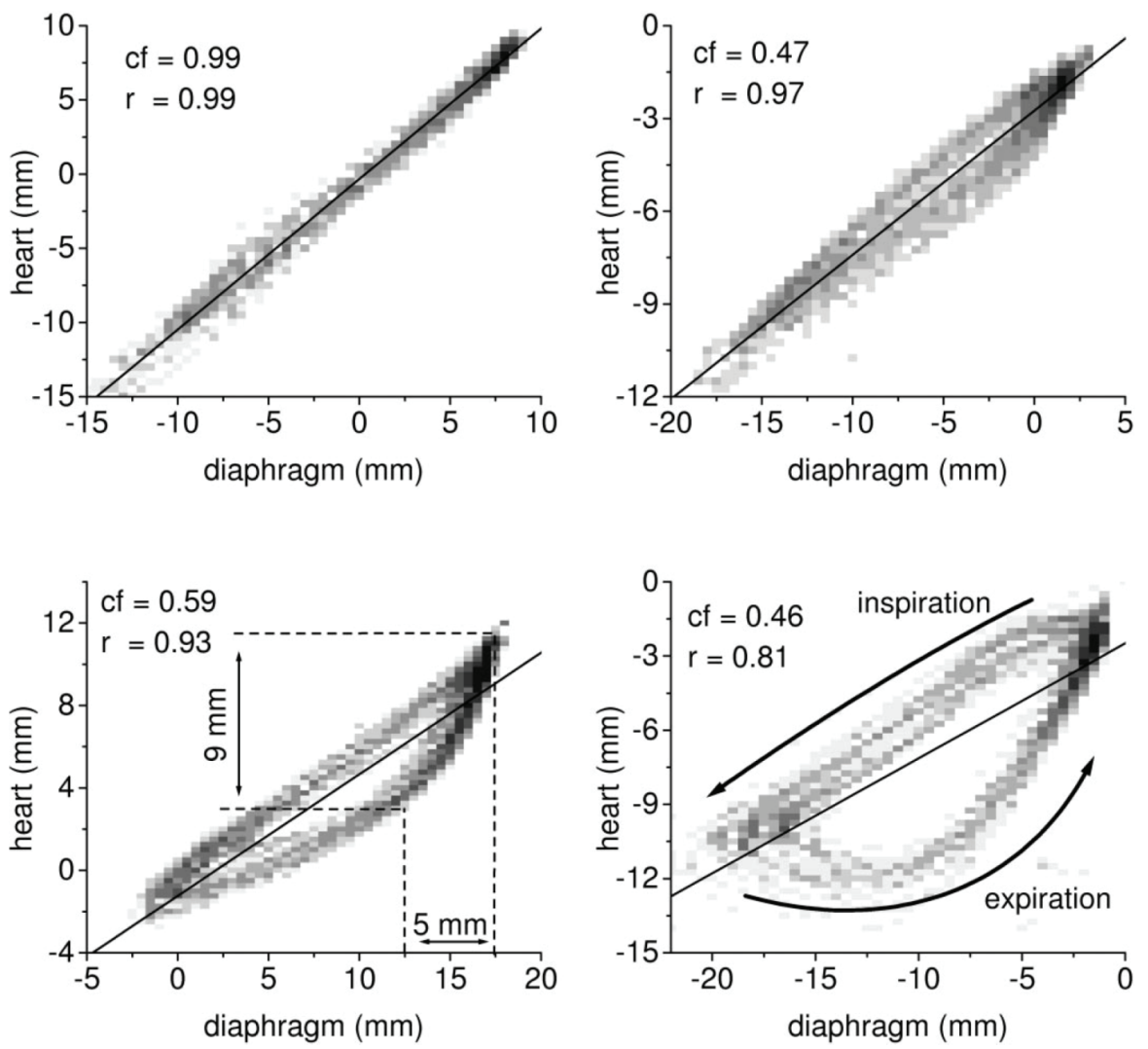

FIGURE 3: Histogram plots of cardiac displacement (heart) as a function of respiration-induced diaphragmatic motion (diaphragm) of respiration obtained in four healthy adult volunteers show considerable variability. Dark squares indicate many counts, while lighter squares indicate fewer counts. The straight lines are linear fits to the data [cf = correction factor (slope) sparsity, $r=$ correlation coefficient]. Bottom right: The direction of the hysteresis is always counterclockwise. Bottom left: The dotted lines depict the mapping of a $5 \mathrm{~mm}$ diaphragmatic gating window (horizontal double-headed arrow) onto the corresponding cardiac displacement range (vertical double-headed arrow). (Adapted and reprinted with permission from Ref. 13.)

extent are not easily predictable.

Because bulk motion is usually rigid (i.e., there is no deformation of the object of interest), the motion can be described by six degrees of freedom (three for rotation and three for translation). ${ }^{17}$ This in turn means that a high degree of modeling of the movement is possible and that a complete description of the motion that needs to be corrected for can be provided should these six parameters be adequately determined. Bulk motion compensation schemes are most commonly applied to brainimaging pulse sequences where the coregistration of different parts of k-space needs to be highly accurate; high-resolution structural imaging and functional MRI are example applications.

\section{COMPENSATING FOR CARDIAC MOTION}

\section{A. Triggering on the Electrocardiogram}

High-resolution anatomic cardiac imaging can be performed during one of the previously discussed cardiac rest periods to minimize the effects of cardiac motion during image acquisition. To this end, data is commonly acquired using electrocardio- 


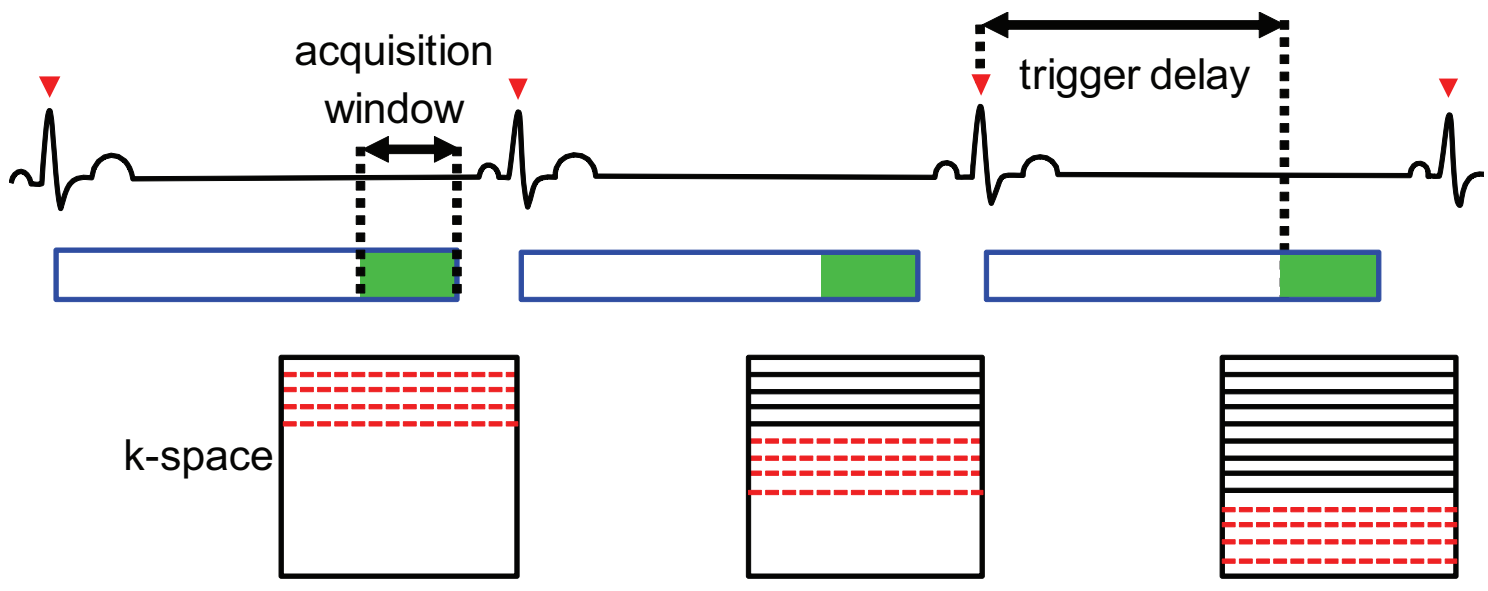

FIGURE 4: Schematic representation of an ECG-triggered segmented k-space acquisition. After detection of the R-wave (small red triangles), a trigger delay follows, after which data are acquired during an acquisition window in the user-selected phase of the cardiac cycle. In this example, the acquisition occurs during the mid-diastolic rest period. Only a segment of k-space is acquired during each acquisition window. Therefore, filling k-space requires acquisition during multiple heartbeats.

gram (ECG) triggering. The main characteristics of an ECG are three so-called waves: a smaller Pwave that is caused by atrial depolarization, a large $\mathrm{R}$-wave that is caused by the depolarization of the ventricles, and a T-wave that is caused by repolarization of the ventricles. ${ }^{18}$ The pulse sequence is synchronized with the R-wave of the ECG and, therefore, data acquisition is synchronized with the heartbeat (Fig. 4). The duration of the rest period is usually not sufficient to collect all the data in $\mathrm{k}$-space. Therefore, data are collected over multiple cardiac cycles in a segmented fashion. The duration of the acquisition window must be long enough to minimize scan time, but short enough to minimize cardiac motion during image data acquisition (Fig. 5).

In prospective cine imaging, theECG-triggered pulse sequence groups a continuous series of segmented acquisitions in bins at a certain time point within the cardiac cycle, and a movie is obtained in which the frames represent the different phases of the cardiac cycle. For retrospective cine imaging, on the other hand, GRE or bSSFP images are continuously acquired and retrospectively binned according to an ECG-related time stamp. ${ }^{19}$ Although it puts higher demands on the stability of the imaging sequence, the latter technique has the dual advantages that it can maintain the steady state for bSSFP imaging while simultaneously allowing access to late diastolic frames in the cardiac cycle, which is often missed by prospective gating (Fig. 6).

In general, the time of the least cardiac motion and thus the trigger delay can be estimated from a patient's heart rate ${ }^{20}$ or visually determined from a cine sequence (Fig. 6). ${ }^{21}$ Alternatively, the use of automated algorithms for the detection of the period of minimal myocardial motion has been suggested..$^{22-24}$ The subsequent scans can then be tailored for data acquisition to occur only during this predefined quiescent period (Fig. 4). Typically, a period of minimal myocardial motion occurs in late diastole, ${ }^{25}$ although end-systolic imaging may be preferable in patients with high heart rates. ${ }^{26,27}$ While ECG triggering is the workhorse of cardiac MRI, it is also applied in peripheral organs where the pulsatility of the blood causes unwanted artifacts, such as in carotid imaging ${ }^{28}$ or cerebral blood flow measurements. ${ }^{29}$

Considerable ECG-signal degradation may occur because of radiofrequency (RF) fields and gradient -switching. Moreover, the so-called magneto- 



FIGURE 5: The effect of ECG triggering on cardiac MR images acquired during free breathing. (a) Axial cardiac image at the level of the left anterior descending (LAD) coronary artery without cardiac motion correction. Several larger structures can still be discerned, but overall the image is blurred and noisy. (b) The same anatomical level as (a), but with ECG triggering. The LAD and other smaller structures can now be discerned, but there is a residual blurriness in the image due to the lack of compensation for respiration motion.

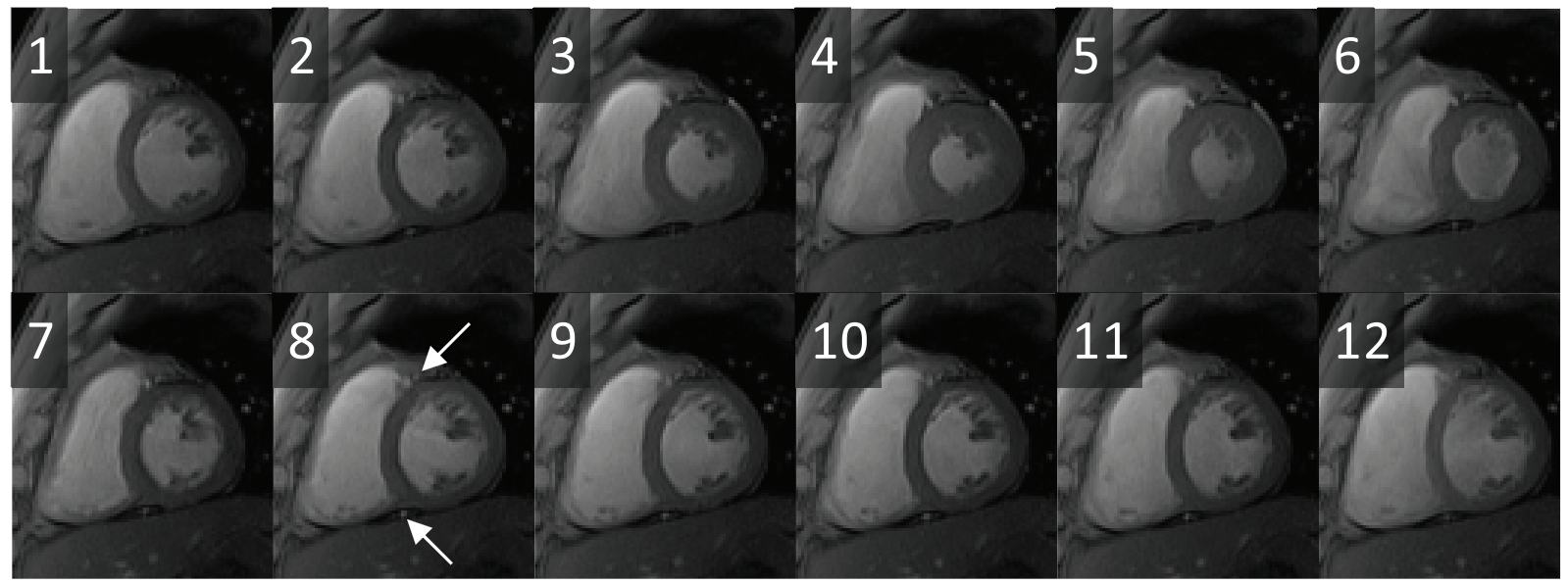

FIGURE 6: A series of 12 out of 24 retrospectively reconstructed cine frames of the heart in a short axis orientation throughout the cardiac cycle of a healthy young volunteer (systole, frames 1-5; diastole, frames 6-12). After the R-wave, the heart quickly contracts during systole, remains relatively quiescent during one or two frames (frames 4-5) at end systole before filling occurs in diastole. The heart also remains relatively quiescent during mid-diastole (frames 9-11). The LAD and RCA can be observed as bright dots above and below the myocardium in the frames where they are at rest (arrows).

hydrodynamic effect creates an artifactual voltage overlaid on the T-wave of the ECG. This artifactual augmentation of the T-wave may frequently mislead the R-wave detection algorithm to trigger the acquisition on the T-wave instead of the R-wave, which results in serious artifacts on cardiac MR images that are acquired with segmented $\mathrm{k}$-space acquisition techniques. Because this ECG artifact increases with magnetic field strength, this presents a major challenge at $3 \mathrm{~T}$ and beyond. Howev- 

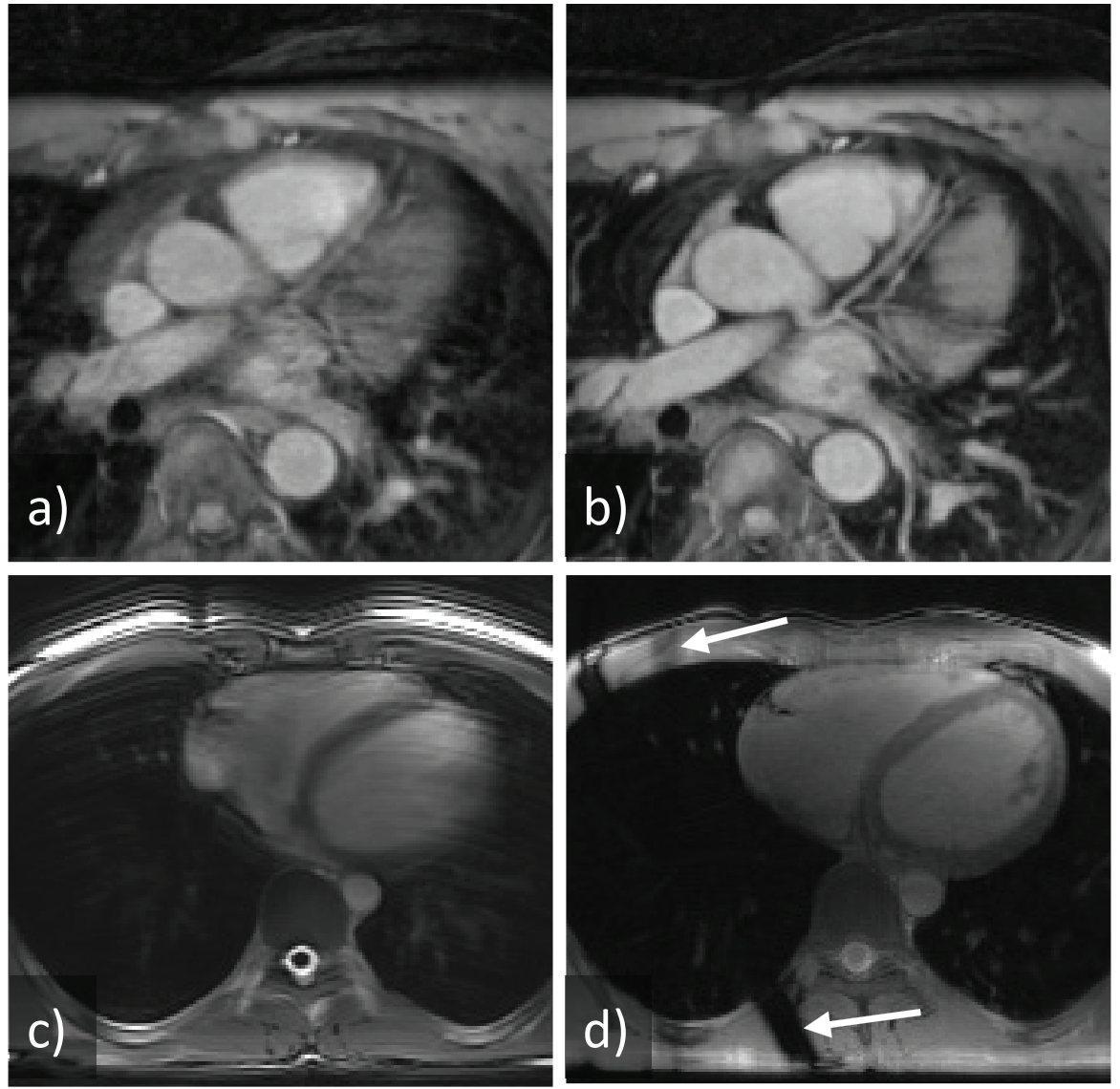

FIGURE 7: The effect of diaphragmatic NAV gating with slice tracking on axial cardiac MR images. (a) The same ECG-gated, free-breathing image as in Fig. 5(b). Blurring distorts the details of the finer structures. (b) Again, the same anatomical level, but now with both ECG triggering and NAV gating. All structures are better defined and the LAD can clearly be discerned. (c) Axial cardiac image at mid-ventricular level without respiratory motion correction. The heart is again blurred and only large structures can be discerned. Ghosting artifacts of the subcutaneous fat can be observed throughout the heart. (d) The same anatomical level as (c), but with NAV gating. The cardiac structures are clearly defined. Conversely, the crossing 90 deg and 180 deg slices of the NAV can be observed at the level of the right lung (arrows).

er, by analyzing the ECG vector in $3 \mathrm{D}$ space, ${ }^{30}$ the true T-wave can be separated from an artifactual T-wave augmentation, and more reliable R-wave detection has shown to be feasible even at higher magnetic field strengths. ${ }^{31}$.

\section{B. Alternative Cardiac Trigger Sources}

As an alternative to the ECG, several external triggering sources have been described, such as pulse oximetry, ${ }^{32}$ the phonocardiogram, ${ }^{33}$ laser Doppler velocimetry, and plethysmograph peripheral pulse monitoring. ${ }^{34}$ In recent times, moreover, self-gating techniques have also emerged. Here, motion information is directly extracted from parts of the acquired image data themselves. ${ }^{35}$

\section{Real-Time Imaging}

Recent advances in hardware and software also 
allow image acquisition in real time, thus avoiding any sort of triggering. The use of short repetition times, radial k-space sampling trajectories and nonlinear reconstruction ${ }^{36,37}$ enables the acquisition of one image in only a few microseconds. In this manner, a complete set of cine images can be obtained in one heartbeat. As always, compromises between temporal resolution, signal-to-noise ratio (SNR), and spatial resolution have to be considered. These techniques are therefore mostly suitable for applications where a high spatial or temporal resolution is not mandatory, and are often a most valuable alternative in patients with arrhythmias or frequent ectopic beats.

\section{COMPENSATING FOR RESPIRATORY MOTION}

\section{A. Breath Holding}

One of the simplest techniques for compensating for respiratory motion is breath holding $(\mathrm{BH})$. Using this approach, the acquisition of an image is performed while the subject is asked to hold his or her breath for 10-30 s. BH approaches offer the advantage of rapid imaging, and are technically easy to implement in compliant subjects. If the acquisition takes too long to fit within a single $\mathrm{BH}$, it is possible to use multiple $\mathrm{BHs}$ and to acquire segments of k-space. ${ }^{38}$ This is, for example, highly suited for lung imaging with hyperpolarized xenon, since the hyperpolarized signal will have decayed after each $\mathrm{BH} .{ }^{39}$ However, due to the relatively poor reproducibility of sequential end-expiratory $\mathrm{BH}$ positions, misregistration errors can be encountered..$^{40,41}$ Furthermore, major patient and operator involvement has to be considered if serial $\mathrm{BHs}$ are required. Alternative $\mathrm{BH}$ techniques that extended the duration of a $\mathrm{BH}$ using supplementary oxygen or hyperventilation have also been described. ${ }^{42}$ However, all these schemes require patient cooperation, and are not always feasible for clinical use. Furthermore, it has also been demonstrated that hypocapnia affects cardiovascular activity ${ }^{43}$ and that residual respiratory motion is not completely suppressed. Diaphragmatic drift is, in fact, also frequently observed during prolonged phases of $\mathrm{BH}^{44}$ and adversely affects image quality.

\section{B. Respiratory Navigators}

As discussed above, the respiratory-induced motion extends in three dimensions, but the SI displacement is the largest component, indicating that the motion can be compensated for to a large degree with a 1D measure. For this reason, most gating approaches measure the SI displacement of the right hemidiaphragm as introduced by Ehman



a)

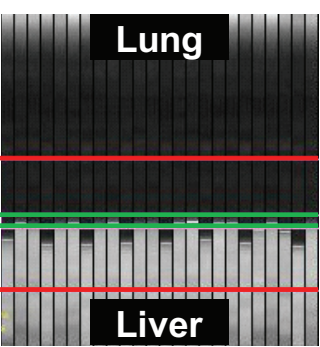

b)

FIGURE 8: Schematic for respiratory motion suppression using navigator gating. (a) When the diaphragmatic interface detected by the navigator (light blue rectangles) falls in the predefined gating window (green lines), the data segment (dark blue rectangles) that is acquired immediately after the navigator is accepted for image reconstruction; otherwise it is rejected and remeasured in the subsequent cardiac cycle. (b) Navigator display as visualized on the console of the system. Time is on the $x$-axis and the navigator-detected lung-liver interface signal profile is displayed on the $y$-axis. When the interface falls within the two green lines, data are accepted. The two red lines indicate the region within which the detection algorithm searches for the interface. 
et. al. ${ }^{45}$ Using so-called "navigators" (NAVs), the diaphragmatic SI displacement is monitored in real time prior to image data acquisition through registration of the displacement of the lung-liver interface in 1D profiles (of the lung-liver interface in SI direction). These 1D profiles are generated by means of orthogonal slice-selective $90 \mathrm{deg}$ and 180 deg pulses or, more recently, through the use of 2D selective (pencil beam) excitations (Fig. 7) ${ }^{46}$

The position of the lung-liver interface just before image acquisition is thus obtained, and is used to accept or reject the measured data with respect to a reference state (Fig. 8). The gating window (GW) is defined as the range of NAV-identified interface positions within which subsequently acquired image data (k-space segment) are accepted for reconstruction. Conversely, if the NAV-identified interface position falls outside of that range or gating window, the image data are rejected, and the k-space segment has to be measured again. Typically, the width of the GW is selected by the user and is $3-8 \mathrm{~mm}$, while its position is either detected by automated algorithms or by the user as well. The position of the GW is often set around the end-expiratory level of the respiratory curve, since it corresponds to a period of slow respiratory motion and the end-expiratory position has a relatively high reproducibility.

The displacement measure obtained with the NAV can also be used to perform prospective motion correction using slice tracking: here, the position of the acquisition volume is adjusted according to the actual position of the NAV interface within the GW (Fig. 8). Using this technique, the residual respiration-induced displacement of the coronaries within the gating window can be accounted for, and respiratory artifacts can be further reduced. This technique was first developed with multiple guided $\mathrm{BHs}^{47}$, and then extended to free breathing. ${ }^{46}$ For this approach, the use of a motion model is required to properly map the diaphragmatic motion to that of the heart. ${ }^{11}$ The earlier-mentioned correction factors for RCA and LAD motion relative to diaphragmatic motion that were reported by Taylor et al. ${ }^{12}$ are commonly used as correction factors for the slice-tracking technique..$^{47,48}$
Using slice tracking, a higher accuracy of the imaged slice position can be obtained relative to the object of interest. Simultaneously, this improved accuracy enables the use of larger gating windows, with a resultant reduction in scanning time. It has to be considered, however, that the relationship between diaphragmatic and heart displacement is not linear, and the use of larger gating windows may therefore lead to additional image artifacts. Limitations of the technique may also be related to hysteresis, as discussed above. However, if the gating window is chosen sufficiently small, such problems can be avoided to a large degree. This type of NAV technology has not only been applied to the heart, but also to other organs including the intestines, kidneys, ${ }^{49}$ and lungs. ${ }^{50}$

The use of a left ventricular NAV ${ }^{51}$ theoretically helps to avoid the need for the above-described motion model that maps the diaphragmatic to the cardiac displacement, and hysteresis may no longer be an issue. However, and despite the theoretical advantage of a left ventricular NAV, to date, there is no study that suggests that a cardiac NAV leads to superior image quality when compared to data acquired with its diaphragmatic counterpart. ${ }^{52}$ In order to account for the nonrigid nature of the respiratory-induced motion of the heart, nonrigid transformations have also been exploited as part of motion compensation strategies in free-breathing, high-resolution cardiovascular MRI. ${ }^{14,15}$ To this end, low-spatial resolution prescans are performed prior to the actual scan and used for parameter estimation of an affine motion model. At the end of this preparatory phase, a motion vector field of the heart is thus obtained, and its parameters are used to correct respiratory-induced deformation of the cardiac anatomy in high-resolution NAV-gated scans. Here, the advantage is that nonrigid motion is taken into account, and a wider gating window can be allowed (20 mm versus a regular $5 \mathrm{~mm}$, for example), which substantially increases scan efficiency ${ }^{53,54}$. However, while very sophisticated and appealing, an advantage in image quality has not been documented in patients with this technique to our knowledge.

An alternative approach includes the frequen- 

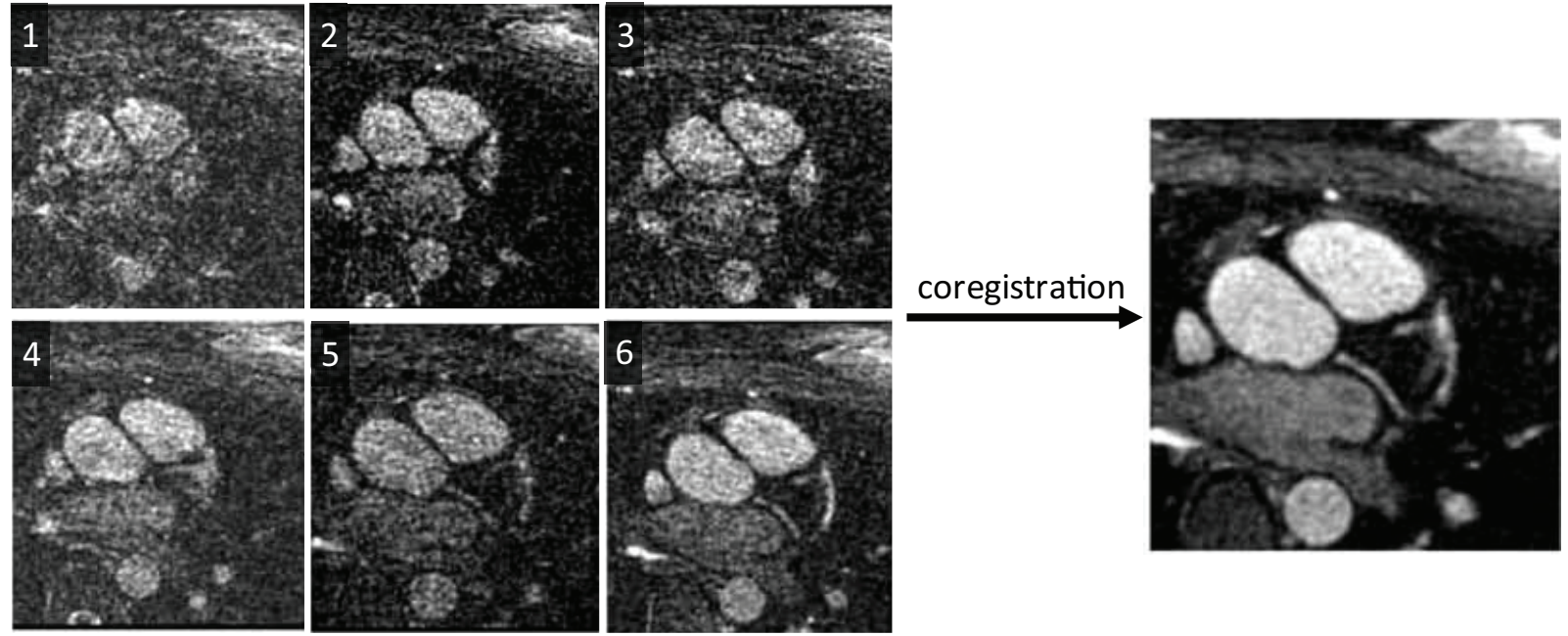

FIGURE 9: Respiratory motion compensation through the use of subimages in an axial slice that contains a portion of the left circumflex (LCX) coronal artery in a healthy volunteer. The acquired data are retrospectively sorted into bins 1-6 according to the cardiac phase at the time of acquisition, after which a subimage is reconstructed from each of these bins (the images on the left). Differences in the subimages due to respiration can be observed. All subimages are then coregistered with bin 1 through affine transformation and summed, resulting in the sharp, highSNR image on the right. (Adapted and reprinted with permission from Ref. 78.)

cy-selective and exclusive excitation of the epicardial fat (which surrounds and moves together with the coronary arteries) using so-called fat-navigator echoes. This fat NAV is also placed just before the image acquisition and collects multiple radial spokes. Here, the respiratory-induced motion is estimated directly from the object of interest, and the data of Nguyen et al. suggest a higher image quality and scan efficiency with respect to conventional diaphragmatic NAVs. ${ }^{55-57}$ Epicardial fat NAVs have also successfully been used in coronary vessel wall imaging. To this end, a beat-tobeat respiratory motion compensation framework was established by acquiring $3 \mathrm{D}$ fat-selective, lowresolution images in each cardiac cycle before the acquisition of the segment that could be correlated retrospectively to estimate the respiratory motion pattern used for final motion correction and reconstruction of a fully sampled spiral data set. ${ }^{58,59} \mathrm{An}-$ other alternative respiration motion compensation technique was proposed by Oshinski et al., who used the signal of respiratory bellows and only accepted acquired data when the spirogram indicated an end-expiration point in the respiratory cycle. ${ }^{60}$
Clearly, the need for imaging during both a short time window in the cardiac cycle and at end expiration decreases the scan efficiency for coronary imaging dramatically. During free-breathing, navigator-gated and -corrected 3D whole-heart coronary MRA that lasts $10 \mathrm{~min}$, a mere $10 \mathrm{~s}$ are used for data collection or signal sampling. This discrepancy leads to a $<2 \%$ scan efficiency for contemporary methods. This example illustrates both the need and the potential for new methods with dramatically improved scan efficiency.

\section{Self-Navigated Approaches}

In direct response to the above need, respiratory motion compensation techniques that directly extract motion parameters from the imaging data themselves rather than from NAVs have recently been developed. These methods that also do not use external devices are called self-navigated approaches. This has mostly been applied to cardiac MRI, and first applications were reported for cine imaging. ${ }^{61}$

When compared to more conventional NAVs, 
self-navigation affords a series of theoretical advantages. First, respiratory motion-induced displacement of the heart is directly obtained from the object of interest, while avoiding indirect measurements or models that relate motion of the diaphragm to that of the heart. Second, there no longer exists a delay between NAVs and imaging, which critically supports imaging at higher spatial resolution. ${ }^{62}$ Third, scout scanning for NAV placement can be avoided. And finally, the scan efficiency is substantially improved.

One category of self-navigated approaches is based on the oversampling of $\mathrm{k}$-space centerline (KCL); in the work of Kim et al. ${ }^{63}$ the information obtained from these profiles was initially used to extract a cardiac and respiratory gating signal in order to perform gating-free acquisitions of the heart and later also found application in abdominal imaging with the work of Brau et al. ${ }^{64}$ More recently, the method has been successfully applied to high-resolution coronary angiography. ${ }^{65}$

In KCL-oversampled self-navigation, the displacement relative to a reference position is computed off line (retrospectively), meaning that the acquisition can be performed with near- $100 \%$ scan efficiency and thus results in a direct motion estimation of the heart itself as well as a substantial decrease in scan time. The method exploits the advantageous motion robustness of the radial sampling scheme, and performs respiratory motion suppression with a simple rigid-body motion correction. There are, however, several drawbacks to using this technique. Similar to regular NAV, the KCLs do not provide information about motion occurring in perpendicular directions. Furthermore, when using KCLs to estimate the position of the heart, it is assumed that other background structures do not influence this estimation. In practice, however, these background structures are static objects that do not vary their position over different central echoes such as the heart does; their presence can thus bias the displacement estimation. In order to cope with this drawback, Lai et al. ${ }^{66}$ proposed using the least-squares error between the KCLs to extract the motion. In further work, the same group improved the suppression of background structures by also acquiring a second anterior-posterior projection (prior to high-spatial resolution data acquisition) from which they extracted the in-plane motion, ${ }^{67}$ while they later extended this translation correction to the third dimension. ${ }^{68}$ KCLs were also exploited in cine imaging to perform real-time respiratory self-gating for whole-heart cine $\mathrm{MRI}^{69}$ and were filtered in real time to extract cardiac and
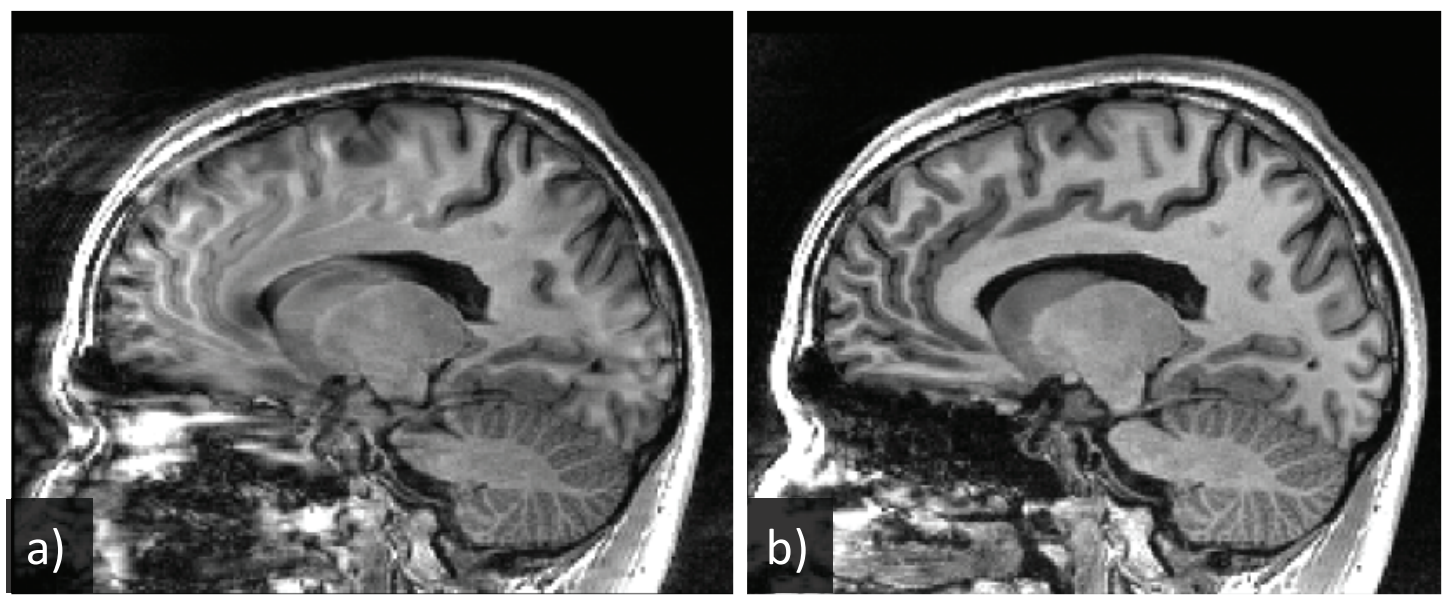

FIGURE 10: The effect of bulk motion on brain MR images. (a) Image without motion correction. Ghosting artifacts can be observed in front of the head, while the nasal cavity is highly distorted and the gray and white matter in the cortex are blurred. (b) The same slice with interscan motion correction. The gray and white matter in the cortex are now more sharply delineated. 


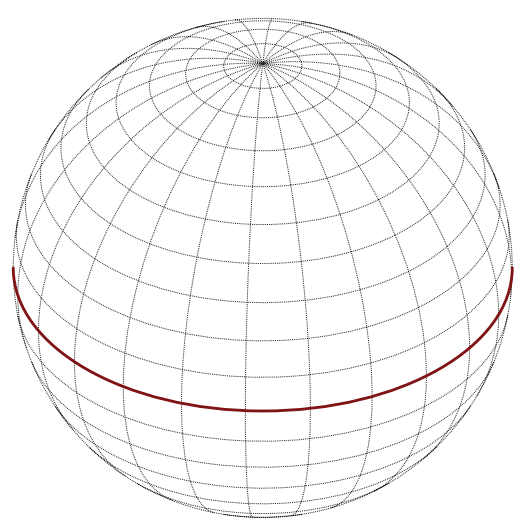

a)

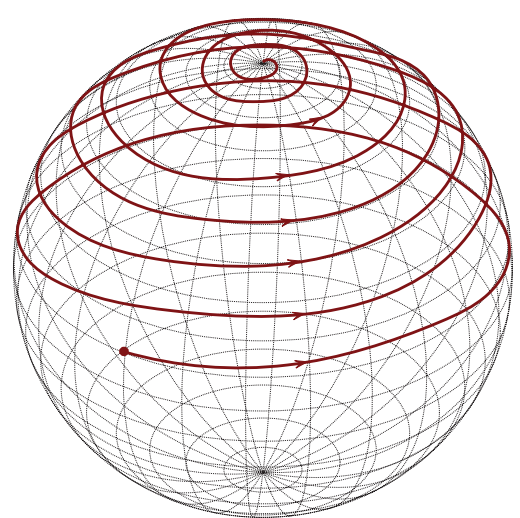

b)



c)

FIGURE 11: K-space trajectories of different navigator techniques. (a) The orbital (ONAV) readout, a, is able to detect translational and rotational in-plane bulk motion. (b) To also take into account movements orthogonal to the image plane, this is extended to spherical trajectory (SNAV). (c) The cloverleaf technique is able to detect 3D translation and rotation very rapidly; in addition, frequency shifts can be derived from the k-space center, which is sampled three times. The small "loopings" at the end of the linear segments are added to reduce the maximal gradient slew rates.

respiratory patterns to perform fully gatingfree dynamic acquisitions. ${ }^{70-72}$

A second self-navigation technique involves the reconstruction of low-resolution images from the fully sampled k-space center in radial cardiac acquisitions $^{61}$ or PROPELLER to compensate for bulk motion occurring in brain imaging. ${ }^{73}$ In coronary MRA, low-resolution images were used for respiratory motion compensation in two dimensions (SI and AP). ${ }^{74}$

Another method to obtain motion information from the original MR data consists of reconstructing data segments that belong to the final image. A series of aliased images that are heavily undersampled are thus obtained, but these can be used for motion detection by applying a motion-tracking method such as image coregistration or cross-correlation. In the case of ECG-gated cardiac MRI, these aliased images, also called subimages, can be obtained by reconstructing each data segment independently and performing a beat-to-beat motion detection or by binning together segment data that belong to the same respiratory phase (Fig. 9). Non-Cartesian sampling methods are well suited for this type of self-navigation: subimages have been reconstructed from interleaved $2 \mathrm{D}$ radial acquisitions to compensate for motion in knee imag- ing $^{75}$ and cardiac imaging ${ }^{76}$, while undersampled subimages from spiral interleaves ${ }^{77}$ and 3D radial sampling patterns ${ }^{78}$ for free-breathing, whole-heart coronary angiography have also been described.

Furthermore, as was proposed by McLeish et al., ${ }^{14}$ respiratory motion can also be compensated with nonrigid transformations that better model the physiological motion. This has been applied to delayed enhanced cardiac MRI, ${ }^{79}$ as well as to wholeheart coronary $\mathrm{MRA}^{80}$ to our knowledge.

\section{COMPENSATING FOR BULK MOTION}

The goal of bulk motion compensation can be divided into two types: intrascan compensations, which compensate for motion within one readout, and interscan compensations, which compensate for motion among readouts. Intrascan compensation techniques detect changes within a single kspace line or echo planar imaging shot and direct the pulse sequence to reacquire the line or shot in question if motion was detected. However, since most modern pulse sequences have very short acquisition times, intrascan motion compensation is usually safely ignored.

Interscan motion compensation techniques can either be used to indicate that a k-space segment 
should be reacquired when motion is detected or, since the exact rotational and translational motion can quite accurately be deduced, to adapt the pulse sequence such that it still acquires the original volume of interest (Fig. 10).

\section{A. Bulk Motion Navigators}

\section{One- and Two-Dimensional Navigators}

Similar to the NAVs discussed for respiratory motion compensation, NAVs can also be used for bulk motion compensation. Since most noncardiac acquisition is not segmented, a NAV module will usually need to be placed within the pulse sequence, i.e., within each repetition time. However, since bulk motion can be completely described by six degrees of freedom, 2D and 3D NAVs that can measure and compensate for movements within minimal time have been developed.

A relatively straightforward implementation is often used in carotid imaging, where swallowing can corrupt the acquisition of high-resolution images of atherosclerotic plaque at the level of the bifurcations. To prevent this, a $1 \mathrm{D}$ NAV is placed on the back of the tongue; ${ }^{28}$ when a nonstandard NAV position is detected, the corresponding data segment is rejected for reconstruction and will be acquired again later.

After the introduction of a 1D NAV for respiration as mentioned earlier ${ }^{45}$, a small phase offset was added by Kadah et al. ${ }^{81}$ to obtain a 2D-sensitive NAV, which they named a floating NAV (FNAV) echo. While its acquisition is quite fast and uses minimal signal, this technique is however only capable of measuring translation. Rotation can also be measured with the orbital navigator (ONAV $)^{82}$. Here, a circular trajectory in k-space is acquired using two simultaneous sinusoid gradients, leading to a fully 2D NAV [Fig. 11(a)]. After acquiring a reference data set, both the rotation and translation can be measured with an accuracy that depends on the diameter of the circle in k-space, which is competing with the ONAV duration. The main disadvantage of the technique is that it only detects in-plane motion, albeit in a short time of $8.2 \mathrm{~ms}$.
This idea was then expanded to three dimensions by Ward et al. ${ }^{83}$ by simply acquiring three orthogonal ONAVs, thus obtaining 3D motion detection at the expense of a time increase to $160 \mathrm{~ms}$ for acquisition and analysis.

\section{Three-Dimensional Navigators}

The first NAV to use a 3D trajectory through kspace was the spherical navigator (SNAV), which consisted of two helical spiral trajectories that are again compared to a reference scan with a precision of $0.4 \mathrm{~mm}$ and $0.26 \mathrm{deg}^{84}$ [Fig 11(b)]. This technique was further optimized in subsequent publications ${ }^{85}$, until a NAV that only scans the "polar caps," dubbed pNAV, reduced the acquisition time to $100 \mathrm{~ms}^{86}$

A different optimization step was performed to obtain so-called cloverleaf navigators. ${ }^{87}$ Here, three orthogonal partial ONAVs are used in combination with three orthogonal linear NAVs to obtain a 3D description of motion [Fig. 11(c)]. This very short k-space trajectory lasts only $4.2 \mathrm{~ms}$ and could therefore be implemented before and after 3D segmented k-space gradient echo imaging. To account for unknown k-space content beyond the trajectory, a 12 s prescan map was utilized.

3D NAVs are of course not limited to k-space only. In the case of fast 2D echo planar images (EPIs), the entire image can be used as a NAV, ${ }^{88}$ which leads to model-less self-navigation as described before.

\section{FID Navigators}

Since it is essentially a free source of signal, the free induction decay (FID) or DC signal of an imaging sequence has recently attracted considerable attention for its use in motion correction. The FID is assumed to be constant once the system is in steady state, and will change only when the effective magnetic field changes. In turn, this means that it will change if the magnetic field is changed due to displacement of the object in a gradient field. ${ }^{89}$ Brau and Brittain expanded this to quantify motion in a $2 \mathrm{D}$ plane by sampling four points in 20 
$\mu$ s,(Ref. 90) and showed good agreement between the FID NAV motion estimation and the signals of the respiration bellows and peripheral pulse. Kober et al. then expanded the technique to make use of RF coil arrays, where multiple FIDs with different spatial sources (the individual coil elements) are collected at the same time, thus providing a more detailed description of the motion..$^{91,92}$

\section{B. External Tracking}

As an alternative to adding NAV modules to the pulse sequence, which requires extra scan time and often increases SAR deposition, several groups have proposed using a setup of external devices to track the bulk motion of the patient. These devices couple any detected motion back to the MR scanner for prospective correction. Some of the external tracking strategies that have been proposed include using ultrasound guidance, ${ }^{93}$ ultrawide band radar, ${ }^{94}$ infrared tracking devices, ${ }^{95}$ gradientinduced signal in electrodes, ${ }^{96}$ active microcoils, ${ }^{97}$ or spectroscopy to determine the angle of rotation of a crystal containing deuterium. ${ }^{98}$ Although these approaches may allow for real-time motion correction by prospectively adjusting magnetic field gradients and RF pulses, their added complexity may be a significant disadvantage.

While none of these interesting techniques have achieved widespread clinical acceptance yet, optical tracking methods (visual or infrared) remain the most active areas of research. Optical methods use markers that are either attached directly to the subject or, more commonly, via a rigid prosthetic. Cameras track the change in position of these markers, transmitting positional and/or angular data (with the previously mentioned six degrees of freedom) to a processing module, which adjusts scanning parameters accordingly. ${ }^{99}$

\section{SUPPORTING IMPROVEMENTS}

In addition to the motion correction and compensation schemes discussed above, there are several pulse sequence elements that do not compensate for motion per se, but significantly improve mo- tion suppression. Since they are easy to overlook, a short overview will be given here.

\section{A. Cancellation of Unwanted Signal}

\section{Fat Saturation}

K-space-based compensation strategies often make use of motion-induced frequency or phase shifts. As such, off-resonant tissue components in the FOV can impede their success. It is thus useful to suppress the proton signal from adipose tissue (fat), which has a chemical shift of $3.5 \mathrm{ppm}$ $(\sim 220 \mathrm{~Hz}$ at $1.5 \mathrm{~T})$. This suppression is commonly achieved using one of three methods: ${ }^{100}$ inversionrecovery imaging (e.g., STIR), opposed-phase imaging, or, most commonly, frequency-selective fat saturation. Furthermore, and especially in the case of slice tracking, the imaged volume remains stationary relative to the object of interest. However, this makes the chest wall appear as a moving object. Therefore, motion artifacts that originate from the chest wall are expected and are even amplified if there is a strong signal from nonsuppressed subcutaneous fat. Therefore, adequate fat suppression may help minimize motion artifacts.

\section{Saturation Bands}

In addition to exploiting relaxation or frequency differences for fat suppression, it is possible to simply suppress all signals from a given anatomical location. By selectively exciting a slab of tissue and subsequently applying a dephasing gradient, all signals from that area can be spoiled. ${ }^{101} \mathrm{Be}-$ sides improving motion correction strategies, and similar to the fat saturation described above, this approach helps to minimize motion artifacts from tissue that is nonstationary relative to the imaged slice position.

\section{Local Excitation}

Correcting for multiple sources of independent motion, such as in free-breathing cardiac imaging, for example, makes it difficult to use many of the 
correction strategies described earlier most effectively. The rigid-body approximation fails in these cases, which results in residual motion artifacts even after correction. Gating thus becomes mandatory. An alternative approach is to reduce the signal from unwanted tissue by using spatially selective excitation. ${ }^{102}$

Conventional slice selection uses a sinc-shaped RF pulse, applied during a constant gradient. Conversely, inner volume approaches ${ }^{103}$ may be used to minimize spurious signals from outside the area of interest, or a cylinder may be excited by using a so-called jinc-shaped RF pulse that is applied during a spiral gradient. In fact, a variety of excitation geometries are possible and can be designed using, for example, the Shinnar-LeRoux algorithm described by Pauly et al. ${ }^{104}$ Using such selective excitation reduces the k-space contribution of unwanted tissues, allowing for more accurate motion correction strategies. ${ }^{105}$

\section{B. Compressed Sensing}

There is substantial research effort directed toward methods aimed at reducing the amount of acquired data without degrading image quality in order to reduce the image acquisition time and thus sensitivity to motion. All efforts at this type of reduced (or sparse) data acquisition can be called compressed sensing or compressive sampling (CS). Such developments are inspired by the fact that MRI data are often redundant to a significant degree. This redundancy can be a known or modeled signal property such as a spatial-temporal correlation ${ }^{106-108}$ or a redundancy that is learned and extracted from the data themselves. ${ }^{109-111}$

The underlying principle is that most MR images are sparse in an appropriate transform domain. Angiograms, for example, primarily contain contrast-enhanced blood vessels in a large void, and already look sparse in the image representation. More complex imagery, such as brain images, can be made sparse by transforming the image into another domain such as the wavelet domain. Dynamic MR images are highly compressible as well: the quasi periodicity of heart images, for example, leads to a sparse temporal Fourier transform. The sparsity of MR images in conjunction with the linear encoding inherent to the MR signal acquisition are two key properties that enable CS in MRI and thus enable the reconstruction of an image from $\mathrm{k}$-space samples that do not necessarily fulfill the Nyquist criterion.

CS techniques were initially adopted for static MR imaging by Lustig et al. ${ }^{112}$ A prominent application of these techniques is dynamic imaging of the heart: in this case, a dynamic image of the heart is a composite of several quasi-static images (k-space segments) acquired over time. ${ }^{113,114}$ Here, sparsity can often be found in the evolution of the signal intensity of a given voxel through the frames when this voxel is analyzed in the time-frequency domain. In whole-heart coronary MRA, CS can also be applied to overcome the strict constraints that are set by motion, thus allowing the acquisition of a whole-heart volume in one breath hold, ${ }^{115}$ or it can be used to compensate for respiratory motion in free-breathing acquisitions. ${ }^{16,117}$

\section{SUMMARY}

As described in this article, motion compensation aims to reduce motion artifacts in MR imaging, with the ultimate goal of improving diagnostic image quality. The primary target applications include cardiac imaging and various forms of abdominal imaging. Although the development of novel and advanced motion compensation strategies remains a very active field of research, many highly effective motion suppression techniques have been developed and are already available on commercial MR scanners. However, with the advent of high-field systems and the new promise of higher spatial resolution (secondary to improved $\mathrm{SNR}$ ), it remains to be ensured that the effectiveness and accuracy of the motion suppression accounts for the smaller voxel size. Simultaneously, as both advanced scanner hardware and software support imaging at improved spatial resolution, motion compensation techniques have begun to attract attention for areas outside the heart as well.

At present, an impressive number of novel and 
innovative motion suppression approaches are being published in the leading journals of the field, and improving scan efficiency in high-resolution cardiac MRI is among the main targets. Fortunately, this continuous progress will undoubtedly advance the value of MRI in general.

\section{ACKNOWLEDGMENTS}

The authors thank Gilles Puy for the valuable discussions on compressed sensing.

\section{REFERENCES}

1. Scott AD, Keegan J, Firmin DN. Motion in cardiovascular MR imaging. Radiology. 2009;250(2):331-51.

2. Matter C, Nagel E, Stuber M, Boesiger P, Hess OM. Assessment of systolic and diastolic LV function by MR myocardial tagging. Basic Res Cardiol. 1996;91 Suppl 2:23-8.

3. Zerhouni EA, Parish DM, Rogers WJ, Yang A, Shapiro EP. Human heart: tagging with MR imaging--a method for noninvasive assessment of myocardial motion. Radiology. 1988 Oct;169(1):59-63.

4. Axel L, Goncalves RC, Bloomgarden D. Regional heart wall motion: two-dimensional analysis and functional imaging with MR imaging. Radiology. 1992 Jun;183(3):745-50.

5. Stuber M, Scheidegger MB, Fischer SE, Nagel E, Steinemann F, Hess OM, Boesiger P. Alterations in the local myocardial motion pattern in patients suffering from pressure overload due to aortic stenosis. Circulation. 1999 Jul 27;100(4):361-8.

6. Karwatowski SP, Mohiaddin R, Yang GZ, Firmin DN, Sutton MS, Underwood SR, Longmore DB. Assessment of regional left ventricular long-axis motion with MR velocity mapping in healthy subjects. J Magn Reson Imaging. 1994 Mar-Apr;4(2):151-5.

7. Kvitting JP, Ebbers T, Engvall J, Sutherland GR, Wranne $\mathrm{B}$, Wigstrom L. Three-directional myocardial motion assessed using 3D phase contrast MRI. J Cardiovasc Magn Reson. 2004;6(3):627-36.

8. Petersen SE, Jung BA, Wiesmann F, Selvanayagam JB, Francis JM, Hennig J, Neubauer S, Robson MD. Myocardial tissue phase mapping with cine phase-contrast $\mathrm{mr}$ imaging: regional wall motion analysis in healthy volunteers. Radiology. 2006 Mar;238(3):816-26.

9. Wang Y, Vidan E, Bergman GW. Cardiac motion of coronary arteries: variability in the rest period and implications for coronary MR angiography. Radiology. 1999 Dec;213(3):751-8.

10. Johnson KR, Patel SJ, Whigham A, Hakim A, Pettigrew
RI, Oshinski JN. Three-dimensional, time-resolved motion of the coronary arteries. J Cardiovasc Magn Reson. 2004;6(3):663-73.

11. Wang Y, Riederer SJ, Ehman RL. Respiratory motion of the heart: kinematics and the implications for the spatial resolution in coronary imaging. Magn Reson Med. 1995;33(5):713-9.

12. Taylor AM, Keegan J, Jhooti P, Firmin DN, Pennell DJ. Calculation of a subject-specific adaptive motion-correction factor for improved real-time navigator echo-gated magnetic resonance coronary angiography. J Cardiovasc Magn Reson. 1999;1(2):131-8.

13. Nehrke K, Bornert P, Manke D, Bock JC. Free-breathing cardiac MR imaging: study of implications of respiratory motion-initial results. Radiology. 2001 Sep;220(3):810-5.

14. McLeish K, Hill DL, Atkinson D, Blackall JM, Razavi R. A study of the motion and deformation of the heart due to respiration. IEEE Trans Med Imaging. 2002;21(9):114250.

15. Shechter G, Ozturk C, Resar JR, McVeigh ER. Respiratory motion of the heart from free breathing coronary angiograms. IEEE Trans Med Imaging. 2004;23(8):1046-56.

16. Taylor AM, Jhooti P, Wiesmann F, Keegan J, Firmin DN, Pennell DJ. MR navigator-echo monitoring of temporal changes in diaphragm position: implications for MR coronary angiography. J Magn Reson Imaging. 1997 JulAug;7(4):629-36.

17. Bernstein MA, King KF, Zhou XJ. Basics of physiologic gating, triggering and monitoring. Handbook of MRI pulse sequences. New York: Academic Press; 2004.

18. Einthoven W. Galvanometrische registratie van het menschelijk electrocardiogram. Herinneringsbundel Professor S S Rosenstein. Leiden, the Netherlands: Eduard IJdo; 1902. p. 101-7.

19. Lenz GW, Haacke EM, White RD. Retrospective cardiac gating: a review of technical aspects and future directions. Magn Reson Imaging. 1989 Sep-Oct;7(5):445-55.

20. Kim WY, Stuber M, Kissinger KV, Andersen NT, Manning WJ, Botnar RM. Impact of bulk cardiac motion on right coronary MR angiography and vessel wall imaging. J Magn Reson Imaging. 2001 Oct;14(4):383-90.

21. Stuber M, Botnar RM, Danias PG, Sodickson DK, Kissinger KV, Van Cauteren M, De Becker J, Manning WJ. Double-oblique free-breathing high resolution three-dimensional coronary magnetic resonance angiography. J Am Coll Cardiol. 1999 Aug;34(2):524-31.

22. Wang Y, Grimm RC, Felmlee JP, Riederer SJ, Ehman RL. Algorithms for extracting motion information from navigator echoes. Magn Reson Med. 1996;36(1):117-23.

23. Jahnke C, Paetsch I, Nehrke K, Schnackenburg B, Bornstedt A, Gebker R, Fleck E, Nagel E. A new approach for rapid assessment of the cardiac rest period for coronary MRA. J Cardiovasc Magn Reson. 2005;7(2):395-9. 
24. Ustun A, Desai M, Abd-Elmoniem KZ, Schar M, Stuber M. Automated identification of minimal myocardial motion for improved image quality on MR angiography at 3 T. AJR Am J Roentgenol. 2007 Mar;188(3):W283-90.

25. Hofman MB, Wickline SA, Lorenz CH. Quantification of in-plane motion of the coronary arteries during the cardiac cycle: implications for acquisition window duration for MR flow quantification. J Magn Reson Imaging. 1998;8(3):568-76.

26. Gharib AM, Herzka DA, Ustun AO, Desai MY, Locklin J, Pettigrew RI, Stuber M. Coronary MR angiography at $3 \mathrm{~T}$ during diastole and systole. J Magn Reson Imaging. 2007 Oct;26(4):921-6.

27. Tangcharoen T, Bell A, Hegde S, Hussain T, Beerbaum P, Schaeffter T, et al. Detection of coronary artery anomalies in infants and young children with congenital heart disease by using MR imaging. Radiology. 2011 Apr;259(1):240-7.

28. Crowe LA, Keegan J, Gatehouse PD, Mohiaddin RH, Varghese A, Symmonds K, Cannell TM, Yang GZ, Firmin DN. 3D volume-selective turbo spin echo for carotid artery wall imaging with navigator detection of swallowing. J Magn Reson Imaging. 2005 Oct;22(4):583-8.

29. Enzmann DR, Marks MP, Pelc NJ. Comparison of cerebral artery blood flow measurements with gated cine and ungated phase-contrast techniques. J Magn Reson Imaging. 1993 Sep-Oct;3(5):705-12.

30. Fischer SE, Wickline SA, Lorenz $\mathrm{CH}$. Novel real-time $\mathrm{R}$-wave detection algorithm based on the vectorcardiogram for accurate gated magnetic resonance acquisitions. Magn Reson Med. 1999;42(2):361-70.

31. Stuber M, Botnar RM, Fischer SE, Lamerichs R, Smink J, Harvey P, Manning WJ. Preliminary report on in vivo coronary MRA at 3 Tesla in humans. Magn Reson Med. 2002 Sep;48(3):425-9.

32. Denslow S, Buckles DS. Pulse oximetry-gated acquisition of cardiac MR images in patients with congenital cardiac abnormalities. Am J Roentgenol. 1993;160(4):831-3.

33. Frauenrath T, Hezel F, Renz W, d'Orth Tde G, Dieringer M, von Knobelsdorff-Brenkenhoff F, Prothmann M, Schulz Menger J, Niendorf T. Acoustic cardiac triggering: a practical solution for synchronization and gating of cardiovascular magnetic resonance at 7 Tesla. J Cardiovasc Magn Reson. 2010;12:67-80.

34. Lanzer P BE, Schiller NB, Crooks LE, Arakawa M, Kaufman L, Davis PL, Herfkens R, Lipton MJ, Higgins CB. Cardiac imaging using gated magnetic resonance. Radiology. 1984;150(1):121-7.

35. Crowe ME, Larson AC, Zhang Q, Carr J, White RD, Li D, Simonetti OP. Automated rectilinear self-gated cardiac cine imaging. Magn Reson Med. 2004 Oct;52(4):782-8.

36. Boll DT, Merkle EM, Seaman DM, Gilkeson RC, Larson AP, Simonetti OP, Duerk JL, Lewin JS. Comparison of ECG-gated rectilinear vs. real-time radial K-space sam- pling schemes in cine True-FISP cardiac MRI. J Cardiovasc Magn Reson. 2004;6(4):793-802.

37. Uecker M, Zhang S, Frahm J. Nonlinear inverse reconstruction for real-time MRI of the human heart using undersampled radial FLASH. Magn Reson Med. 2010 Jun;63(6):1456-62.

38. Feinberg DA, Rofsky NM, Johnson G. Multiple breathhold averaging (MBA) method for increased SNR in abdominal MRI. Magn Reson Med. 1995 Dec;34(6):905-9.

39. Patz S, Hersman FW, Muradian I, Hrovat MI, Ruset IC, Ketel S, Jacobson F, Topulos GP, Hatabu H, Butler JP. Hyperpolarized (129)Xe MRI: a viable functional lung imaging modality? Eur J Radiol. 2007 Dec;64(3):33544.

40. Wang Y, Grimm RC, Rossman PJ, Debbins JP, Riederer SJ, Ehman RL. 3D coronary MR angiography in multiple breath-holds using a respiratory feedback monitor. Magn Reson Med. 1995 Jul;34(1):11-6.

41. Liu YL, Riederer SJ, Rossman PJ, Grimm RC, Debbins JP, Ehman RL. A monitoring, feedback, and triggering system for reproducible breath-hold MR imaging. Magn Reson Med. 1993 Oct;30(4):507-11.

42. Danias PG, Stuber M, Botnar RM, Kissinger KV, Chuang ML, Manning WJ. Navigator assessment of breath-hold duration: impact of supplemental oxygen and hyperventilation. Am J Roentgenol. 1998 Aug;171(2):395-7.

43. Raper AJ, Richardson DW, Kontos HA, Patterson JL Jr. Circulatory responses to breath holding in man. J Appl Physiol. 1967;22(2):201-6.

44. Holland AE, Goldfarb JW, Edelman RR. Diaphragmatic and cardiac motion during suspended breathing: preliminary experience and implications for breath-hold MR imaging. Radiology. 1998 Nov;209(2):483-9.

45. Ehman RL, Felmlee JP. Adaptive technique for highdefinition MR imaging of moving structures. Radiology. 1989 Oct;173(1):255-63.

46. Danias PG, McConnell MV, Khasgiwala VC, Chuang ML, Edelman RR, Manning WJ. Prospective navigator correction of image position for coronary MR angiography. Radiology. 1997;203(3):733-6.

47. McConnell MV, Khasgiwala VC, Savord BJ, Chen MH, Chuang ML, Edelman RR, Manning WJ. Prospective adaptive navigator correction for breath-hold MR coronary angiography. Magn Reson Med. 1997;37(1):14852.

48. Weiger M, Bornert P, Proksa R, Schaffter T, Haase A. Motion-adapted gating based on k-space weighting for reduction of respiratory motion artifacts. Magn Reson Med. 1997;38(2):322-33.

49. Attenberger UI, Sourbron SP, Michaely HJ, Reiser MF, Schoenberg SO. Retrospective respiratory triggering renal perfusion MRI. Acta Radiol. 2010 Dec;51(10):116371.

50. Molinari F, Eichinger M, Risse F, Plathow C, Puderbach 
M, Ley S, Herth F, Bonomo L, Kauczor HU, Fink C. Navigator-triggered oxygen-enhanced MRI with simultaneous cardiac and respiratory synchronization for the assessment of interstitial lung disease. J Magn Reson Imaging. 2007 Dec;26(6):1523-9.

51. McConnell MV, Khasgiwala VC, Savord BJ, Chen MH, Chuang ML, Edelman RR, Manning WJ. Comparison of respiratory suppression methods and navigator locations for MR coronary angiography. Am J Roentgenol. 1997;168(5):1369-75.

52. Stuber M, Botnar RM, Spuentrup E, Kissinger KV, Manning WJ. Three-dimensional high-resolution fast spinecho coronary magnetic resonance angiography. Magn Reson Med. 2001;45(2):206-11.

53. Jahnke C, Nehrke K, Paetsch I, Schnackenburg B, Gebker R, Fleck E, Nagel E. Improved bulk myocardial motion suppression for navigator-gated coronary magnetic resonance imaging. J Magn Reson Imaging. 2007 Sep;26(3):780-6.

54. Jahnke C, Paetsch I, Nehrke K, Schnackenburg B, Gebker R, Fleck E, Nagel E. Rapid and complete coronary arterial tree visualization with magnetic resonance imaging: feasibility and diagnostic performance. Eur Heart J. 2005 Nov;26(21):2313-9.

55. Nguyen TD, Nuval A, Mulukutla S, Wang Y. Direct monitoring of coronary artery motion with cardiac fat navigator echoes. Magn Reson Med. 2003 Aug;50(2):235-41.

56. Nguyen TD, Spincemaille P, Prince MR, Wang Y. Cardiac fat navigator-gated steady-state free precession $3 \mathrm{D}$ magnetic resonance angiography of coronary arteries. Magn Reson Med. 2006 Jul;56(1):210-5.

57. Nguyen TD, Spincemaille P, Cham MD, Weinsaft JW, Prince MR, Wang Y. Free-breathing 3D steady-state free precession coronary magnetic resonance angiography: comparison of diaphragm and cardiac fat navigators. J Magn Reson Imaging. 2008 Aug;28(2):509-14.

58. Keegan J, Gatehouse PD, Yang GZ, Firmin DN. Nonmodel-based correction of respiratory motion using beatto-beat 3D spiral fat-selective imaging. J Magn Reson Imaging. 2007 Sep;26(3):624-9.

59. Scott AD, Keegan J, Firmin DN. High-resolution 3D coronary vessel wall imaging with near $100 \%$ respiratory efficiency using epicardial fat tracking: reproducibility and comparison with standard methods. J Magn Reson Imaging. 2011;33(1):77-86.

60. Oshinski JN, Hofland L, Mukundan S, Jr., Dixon WT, Parks WJ, Pettigrew RI. Two-dimensional coronary MR angiography without breath holding. Radiology. 1996 Dec;201(3):737-43.

61. Larson AC, White RD, Laub G, McVeigh ER, Li D, Simonetti OP. Self-gated cardiac cine MRI. Magn Reson Med. 2004;51(1):93-102.

62. Spuentrup E, Manning WJ, Botnar RM, Kissinger KV, Stuber M. Impact of navigator timing on free-breathing submillimeter 3D coronary magnetic resonance angiography. Magn Reson Med. 2002;47(1):196-201.

63. Kim WS, Mun CW, Kim DJ, Cho ZH. Extraction of cardiac and respiratory motion cycles by use of projection data and its applications to NMR imaging. Magn Reson Med. 1990;13(1):25-37.

64. Brau AC, Brittain JH. Generalized self-navigated motion detection technique: Preliminary investigation in abdominal imaging. Magn Reson Med. 2006;55(2):263-70.

65. Stehning C, Bornert P, Nehrke K, Eggers H, Stuber M. Free-breathing whole-heart coronary MRA with 3D radial SSFP and self-navigated image reconstruction. Magn Reson Med. 2005 Aug;54(2):476-80.

66. Lai P, Larson AC, Park J, Carr JC, Li D. Respiratory selfgated four-dimensional coronary MR angiography: a feasibility study. Magn Reson Med. 2008;59(6):1378-85.

67. Lai P, Larson AC, Bi X, Jerecic R, Li D. A dual-projection respiratory self-gating technique for wholeheart coronary MRA. J Magn Reson Imaging. 2008 Sep;28(3):612-20.

68. Lai $\mathrm{P}, \mathrm{Bi} \mathrm{X}$, Jerecic $\mathrm{R}, \mathrm{Li} \mathrm{D}$. A respiratory self-gating technique with $3 \mathrm{D}$-translation compensation for freebreathing whole-heart coronary MRA. Magn Reson Med. 2009 Sep;62(3):731-8.

69. Uribe S, Muthurangu V, Boubertakh R, Schaeffter T, Razavi R, Hill DL, Hansen MS. Whole-heart cine MRI using real-time respiratory self-gating. Magn Reson Med. 2007 Mar;57(3):606-13.

70. Spincemaille P, Nguyen TD, Prince MR, Wang Y. Kalman filtering for real-time navigator processing. Magn Reson Med. 2008 Jul;60(1):158-68.

71. Buehrer M, Curcic J, Boesiger P, Kozerke S. Prospective self-gating for simultaneous compensation of cardiac and respiratory motion. Magn Reson Med. 2008 Sep;60(3):683-90.

72. Manka R, Buehrer M, Boesiger P, Fleck E, Kozerke S. Performance of simultaneous cardiac-respiratory selfgated three-dimensional MR imaging of the heart: initial experience. Radiology. 2010 Jun;255(3):909-16.

73. Pipe JG. Motion correction with PROPELLER MRI: application to head motion and free-breathing cardiac imaging. Magn Reson Med. 1999 Nov;42(5):963-9.

74. Henningsson M, Koken P, Stehning C, Razavi R, Prieto C, Botnar RM. Whole-heart coronary MR angiography with 2D self-navigated image reconstruction. Magn Reson Med. 2012 Feb;67(2):437-45.

75. Schaffter T, Rasche V, Carlsen IC. Motion compensated projection reconstruction. Magn Reson Med. 1999;41(5):954-63.

76. McLeish K, Kozerke S, Crum WR, Hill DL. Free-breathing radial acquisitions of the heart. Magn Reson Med. 2004 Nov;52(5):1127-35.

77. Hardy CJ, Zhao L, Zong X, Saranathan M, Yucel EK. Coronary MR angiography: respiratory motion cor- 
rection with BACSPIN. J Magn Reson Imaging. 2003;17(2):170-6.

78. Bhat H, Ge L, Nielles-Vallespin S, Zuehlsdorff S, Li D. $3 \mathrm{D}$ radial sampling and 3D affine transform-based respiratory motion correction technique for free-breathing whole-heart coronary MRA with 100\% imaging efficiency. Magn Reson Med. 2011;65(5):1269-77.

79. Ledesma-Carbayo MJ, Kellman P, Hsu LY, Arai AE, McVeigh ER. Motion corrected free-breathing delayedenhancement imaging of myocardial infarction using nonrigid registration. J Magn Reson Imaging. 2007 Jul;26(1):184-90.

80. Schmidt JF, Buehrer M, Boesiger P, Kozerke S. Nonrigid retrospective respiratory motion correction in whole-heart coronary MRA. Magn Reson Med. 2011 Dec;66(6):1541-9.

81. Kadah YM, Abaza AA, Fahmy AS, Youssef AB, Heberlein K, Hu XP. Floating navigator echo (FNAV) for inplane 2D translational motion estimation. Magn Reson Med. 2004;51(2):403-7.

82. Fu ZW, Wang Y, Grimm RC, Rossman PJ, Felmlee JP, Riederer SJ, Ehman RL. Orbital navigator echoes for motion measurements in magnetic resonance imaging. Magn Reson Med. 1995 Nov;34(5):746-53.

83. Ward HA, Riederer SJ, Grimm RC, Ehman RL, Felmlee JP, Jack CR Jr. Prospective multiaxial motion correction for fMRI. Magn Reson Med. 2000;43(3):459-69.

84. Welch EB, Manduca A, Grimm RC, Ward HA, Jack CR Jr. Spherical navigator echoes for full 3D rigid body motion measurement in MRI. Magn Reson Med. 2002;47(1):32-41.

85. Petrie DW, Costa AF, Takahashi A, Yen YF, Drangova M. Optimizing spherical navigator echoes for three-dimensional rigid-body motion detection. Magn Reson Med. 2005;53(5):1080-7.

86. Costa AF, Petrie DW, Yen YF, Drangova M. Using the axis of rotation of polar navigator echoes to rapidly measure $3 \mathrm{D}$ rigid-body motion. Magn Reson Med. 2005;53(1):150-8.

87. van der Kouwe AJ, Benner T, Dale AM. Real-time rigid body motion correction and shimming using cloverleaf navigators. Magn Reson Med. 2006 Nov;56(5):1019-32.

88. Thesen S, Heid O, Mueller E, Schad LR. Prospective acquisition correction for head motion with imagebased tracking for real-time fMRI. Magn Reson Med. 2000;44(3):457-65.

89. Pfeuffer J, Van de Moortele PF, Ugurbil K, Hu X, Glover $\mathrm{GH}$. Correction of physiologically induced global offresonance effects in dynamic echo-planar and spiral functional imaging. Magn Reson Med. 2002;47(2):34453.

90. Brau AC, Brittain JH. Generalized self-navigated motion detection technique: Preliminary investigation in abdominal imaging. Magn Reson Med. 2006;55(2):263-70.
91. Kober T, Marques JP, Gruetter R, Krueger G. Head motion detection using FID navigators. Magn Reson Med. 2011 Jul;66(1):135-43.

92. Kober T, Gruetter R, Krueger G. Prospective and retrospective motion correction in diffusion magnetic resonance imaging of the human brain. Neuroimage. 2012 Jan 2;59(1):389-98.

93. Günther M, Feinberg DA. Ultrasound-guided MRI: Preliminary results using a motion phantom. Magn Reson Med. 2004;52(1):27-32.

94. Thiel F, Hein M, Schwarz U, Sachs J, Seifert F. Combining magnetic resonance imaging and ultrawideband radar: A new concept for multimodal biomedical imaging. New York: AIP; 2009.

95. Tremblay M, Tam F, Graham SJ. Retrospective coregistration of functional magnetic resonance imaging data using external monitoring. Magn Reson Med. 2005;53(1):141-9.

96. Vestergaard MB, Schultz J, Turner R, Hanson LG. Motion tracking from gradient induced signals in electrode recordings. Proceedings of the ESMRMB 28th Annual Meeting. Leipzig, Germany; 2011.

97. Ooi MB, Krueger S, Thomas WJ, Swaminathan SV, Brown TR. Prospective real-time correction for arbitrary head motion using active markers. Magn Reson Med. 2009;62(4):943-54.

98. Peshkovsky A, Knuth KH, Helpern JA. Motion correction in MRI using an apparatus for dynamic angular position tracking (ADAPT). Magn Reson Med. 2003;49(1):138-43.

99. Zaitsev M, Dold C, Sakas G, Hennig J, Speck O. Magnetic resonance imaging of freely moving objects: prospective real-time motion correction using an external optical motion tracking system. Neuroimage. 2006 Jul 1;31(3):1038-50.

100. Delfaut EM, Beltran J, Johnson G, Rousseau J, Marchandise X, Cotten A. Fat suppression in MR imaging: Techniques and pitfalls. Radiographics. 1999 March 1, 1999;19(2):373-82.

101. Edelman RR, Atkinson DJ, Silver MS, Loaiza FL, Warren WS. FRODO pulse sequences: a new means of eliminating motion, flow, and wraparound artifacts. Radiology. 1988;166(1 Pt 1):231-6.

102. Pauly JM, Nishimura DG, Macovski A. A k-space analysis of small-tip-angle excitation. J Magn Reson. 1989;81:43-56.

103. Feinberg DA, Hoenninger JC, Crooks LE, Kaufman L, Watts JC, Arakawa M. Inner volume MR imaging: technical concepts and their application. Radiology. 1985 Sep;156(3):743-7.

104. Pauly J, Le Roux P, Nishimura D, Macovski A. Parameter relations for the Shinnar-Le Roux selective excitation pulse design algorithm. IEEE Trans Med Imag. 1991;10:53-65. 
105. Abd-Elmoniem KZ, Barmet C, Stuber M. Free-breathing inner-volume black-blood imaging of the human heart using two-dimensionally selective local excitation at 3T. Magn Reson Med. 2012; In Press.

106. Madore B, Glover GH, Pelc NJ. Unaliasing by fourierencoding the overlaps using the temporal dimension (UNFOLD), applied to cardiac imaging and fMRI. Magn Reson Med. 1999 Nov;42(5):813-28.

107. Willis NP, Bresler Y. Optimal scan for time-varying tomography. II. Efficient design and experimental validation. IEEE Trans Image Process. 1995;4(5):654-66.

108. Parrish T, Hu X. Continuous update with random encoding (CURE): a new strategy for dynamic imaging. Magn Reson Med. 1995;33(3):326-36.

109. Hanson JM, Liang ZP, Magin RL, Duerk JL, Lauterbur PC. A comparison of RIGR and SVD dynamic imaging methods. Magn Reson Med. 1997 Jul;38(1):161-7.

110. Tsao J, Boesiger P, Pruessmann KP. k-t BLAST and k-t SENSE: dynamic MRI with high frame rate exploiting spatiotemporal correlations. Magn Reson Med. 2003 Nov;50(5):1031-42.

111. Mistretta CA, Wieben O, Velikina J, Block W, Perry J, Wu Y, Johnson K, Wu Y. Highly constrained backprojection for time-resolved MRI. Magn Reson Med. 2006;55(1):30-40.

112. Lustig M, Donoho D, Pauly JM. Sparse MRI: The application of compressed sensing for rapid MR imaging.
Magn Reson Med. 2007 Dec;58(6):1182-95.

113. Jung H, Ye JC, Kim EY. Improved k-t BLAST and k-t SENSE using FOCUSS. Phys Med Biol. 2007 Jun 7;52(11):3201-26.

114. Usman M, Prieto C, Schaeffter T, Batchelor PG. k-t Group sparse: a method for accelerating dynamic MRI. Magn Reson Med. 2011 Oct;66(4):1163-76.

115. Santos JM, Cunningham CH, Lustig M, Hargreaves BA, Hu BS, Nishimura DG, Pauly JM. Single breath-hold whole-heart MRA using variable-density spirals at 3T. Magn Reson Med. 2006;55(2):371-9.

116. Akçakaya M, Nam S, Hu P, Moghari MH, Ngo LH, Tarokh V, Manning WJ, Nezafat R. Compressed sensing with wavelet domain dependencies for coronary MRI: a retrospective study. IEEE Trans Med Imaging. 2011;30(5):1090-9.

117. Moghari MH, Akçakaya M, O’Connor A, Basha TA, Casanova M, Stanton D, Goepfert L, Kissinger KV, Goddu B, Chuang ML, Tarokh V, Manning WJ, Nezafat R. Compressed-sensing motion compensation (CosMo): a joint prospective-retrospective respiratory navigator for coronary MRI. Magn Reson Med. 2011 Dec;66(6):167481.

118. Abd-Elmoniem KZ, Osman NF, Prince JL, Stuber M. Three-dimensional magnetic resonance myocardial motion tracking from a single image plane. Magn Reson Med. 2007 Jul;58(1):92-102. 
